\title{
UNA NUEVA ESPECIE DE BERBERIS (BERBERIDACEAE) DE LAS MONTAÑAs del Noroeste de ARgentina y sur DE BoliVia
}

\author{
HUGO AYARDE ${ }^{1}$ y EVA BULACIO 2
}

\begin{abstract}
Resumen: Se describe una nueva especie, Berberis calilehua (Berberidaceae), que habita las montañas húmedas del noroeste de Argentina y sur de Bolivia, por encima de los $3000 \mathrm{~m} \mathrm{s.m.} \mathrm{Esta} \mathrm{especie}$ tiene similitudes morfológicas con las especies andinas de Perú y Bolivia pero difiere de aquellas por combinación de caracteres tales como flores solitarias, fruto con estilo conspicuo, margen foliar espinoso dentado y venación dicotómica abierta. Se incluye una detallada descripción de la entidad propuesta, una clave para diferenciar las especies afines, ilustraciones y fotografías.
\end{abstract}

Palabras clave: Argentina, Berberis calilehua, Bolivia, pastizales montanos, Sierras de Calilegua.

Summary: A new species of Berberis (Berberidaceae) from the mountains of northwestern Argentina and southern Bolivia. In this paper, Berberis calilehua, which inhabits the humid mountains, above 3,000 m asl, is described. This species has morphological similarities with Andean species of Peru and Bolivia but differs from those by combination of characters such as solitary flowers, fruit with conspicuous style, leaf margin toothed spinose, open dichotome venation. A detailed description of the proposed entity, a key differentiating related species, illustrations and photographs are included.

Key words: Argentina, Berberis calilehua, Bolivia, montane grasslands, Sierras of Calilegua.

\section{INTRODUCCIÓN}

En el transcurso de un estudio de la vegetación de las Sierras de Calilegua (Jujuy, Argentina), obtuvimos especímenes de Berberis L. (Berberidaceae) que no pudimos adjudicarlos a ninguna especie conocida de Argentina. Nuevas colectas y observaciones "in situ" en distintas localidades de las mencionadas sierras, consultas a herbarios de referencia de Argentina (CORD, LIL, SI), Bolivia (BOLV, HSB, LPB, USZ) y a herbarios virtuales (BM, GOET, K, MICH, US), sumado a los análisis de rutina de caracteres morfológicos del material revisado, aportaron información suficiente para proponerla como una nueva especie.

\footnotetext{
${ }^{1}$ Instituto de Ecología. Fundación Miguel Lillo, Miguel Lillo 251, 4000 San Miguel de Tucumán, Tucumán, Argentina. hayarde@lillo.org.ar

2 Laboratorio de Taxonomía Fanerogámica. Fundación Miguel Lillo, Miguel Lillo 251, 4000 San Miguel de Tucumán, Tucumán, Argentina. evabulacio@yahoo. com.ar
}

Berberis es un género holártico de distribución mundial, que cuenta con alrededor de 500 especies (Ahrendt, 1961) y que en América se extiende hasta los Andes patagónicos. En Argentina se reconocen 20 especies (Orsi, 1976; Zuloaga \& Morrone, 1999, Zuloaga, et al., 2015), la mayoría distribuidas a lo largo del área andina; 32 en Perú (Liesner, 1993) y 15 para Bolivia (Ulloa Ulloa, 2014), aunque consideramos que el número de especies para este último país debe ser mayor.

En este artículo se da a conocer a Berberis calilehua Ayarde et Bulacio, una especie que crece en pastizales de las montañas del noroeste de Argentina y del sur de Bolivia.

\section{Resultados}

Berberis calilehua Ayarde et Bulacio, sp. nov. TIPO: Argentina. Jujuy, dpto. Valle Grande, Sierras de Calilegua, Cerro Hermoso, 3400 m s.m., 3-X2008, Bulacio \& Ayarde 1296 (holotypus LIL, isotipi CORD, LPB, SI). Figs. 1 y 2. 
Bol. Soc. Argent. Bot. 50 (4) 2015

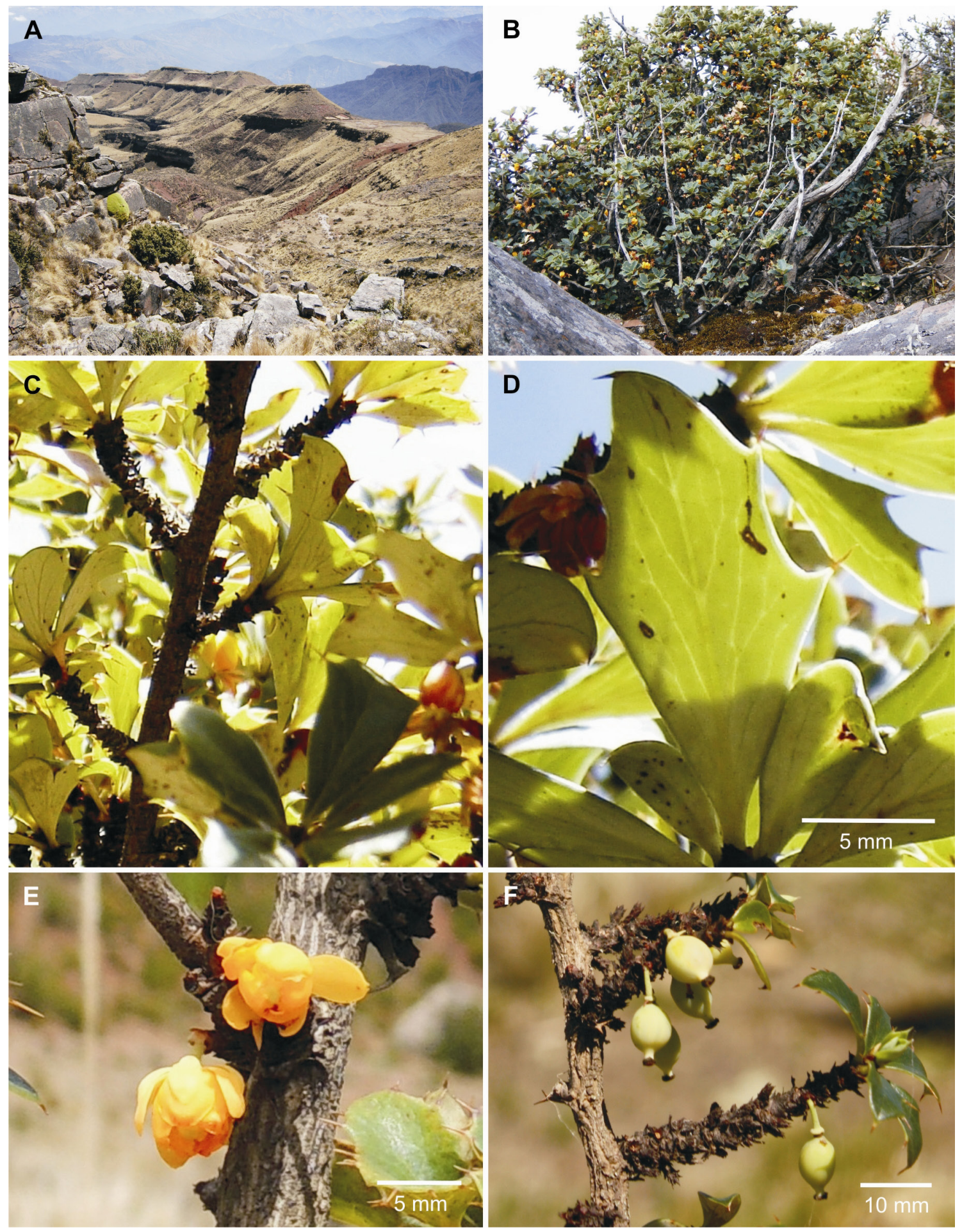

Fig. 1. Berberis calilehua. A: Hábitat (Sierras de Calilegua, Jujuy, Argentina). B: Aspecto de la planta. C: Detalles de ramas principal y secundarias (braquiblastos). D: Detalle de una hoja con nerviación dicotómica abierta y margen con dientes espinosos. E: Flores. F: Frutos inmaduros. 
Hugo Ayarde y Eva Bulacio - Una nueva especie de Berberis de Argentina y Bolivia

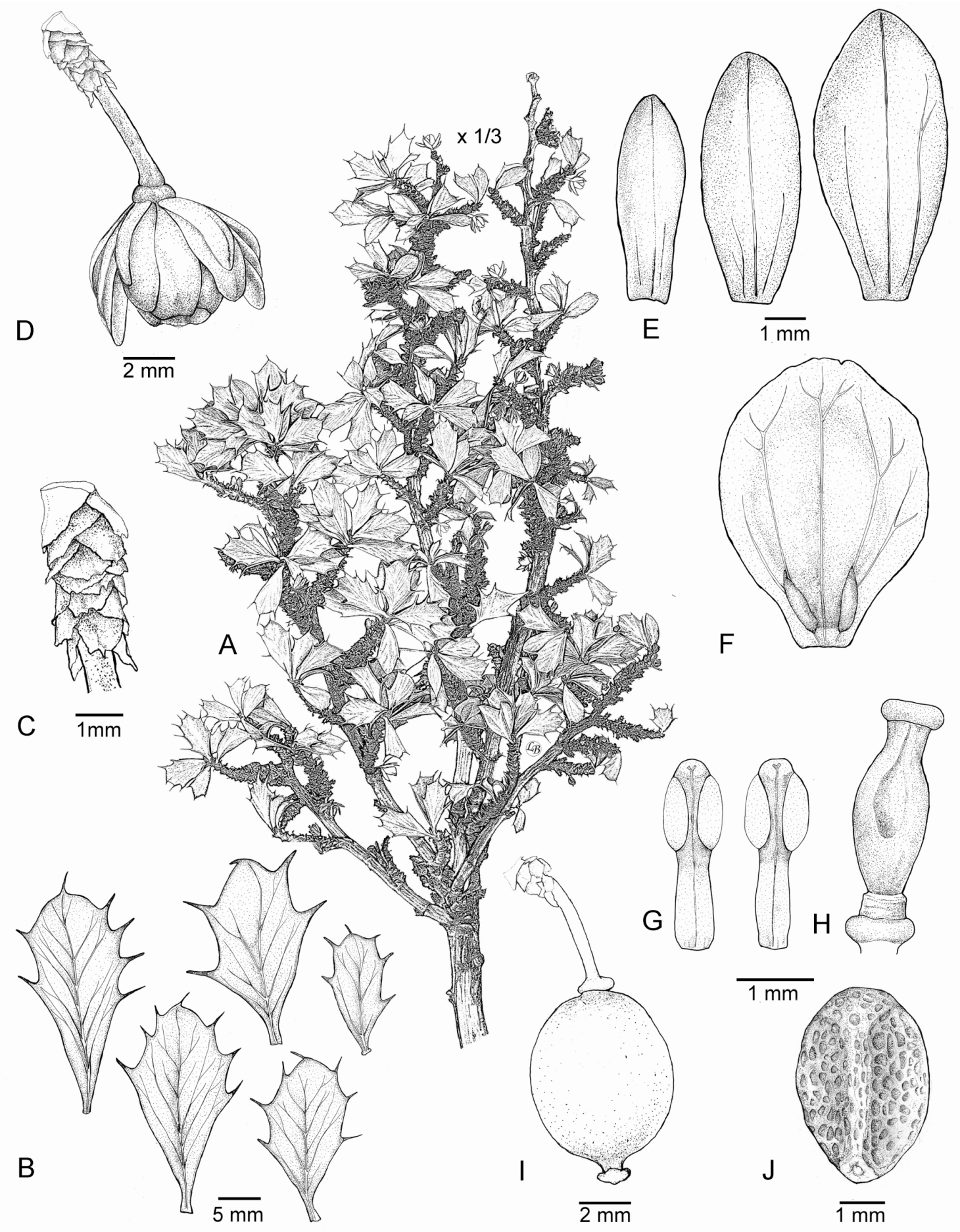

Fig. 2. Berberis calilehua. A: Aspecto de ramas floríferas. B. Hojas. C: Detalle de las escamas de la base del pedúnculo. D: Flor. E: Sépalos. F: Detalle del pétalo con las glándulas basales. G: Estambres. H: Gineceo. I: Fruto. J: Semilla (Bulacio \& Ayarde 1296). 
Frutex globoso $1 \mathrm{~m}$ alta; ramis secunda brevis, apice densius foliata; foliis obovatis, dentato spinosis ad apicem, ramosis, venatione dichotomous cum insertionem acuti; floribus solitariis conspicuous style in fructu.

Arbusto achaparrado, densamente ramificado. Ramas principales rígidas; las jóvenes castañooscuras, verrucoso-pubérulas y de sección más o menos circular; las viejas, grisáceas, surcadas y glabras. Ramas secundarias (braquiblastos) de hasta $50 \mathrm{~mm}$ long. x $6 \mathrm{~mm}$ diám., densamente cubiertas por escamas y bases foliares; escamas triangulares, apiculadas, de 2,2-2,6 mm long. x 2-2,5 mm lat. Espinas, en la base de cada braquiblasto, simples a 5 (-7)-fidas, blandas, caedizas, de 5-15 $\mathrm{mm}$ long. incluida la base ensanchada. Hojas cortamente pecioladas, dispuestas apretadamente en número de 5 a 15 en el ápice de los braquiblastos; láminas glabras, obovadas, de (13-) 15-22 (-25) mm long. x 7-11 $\mathrm{mm}$ lat., ápice obtuso, mucronado y base cuneada; margen engrosado, sinuado, con 2 a 4 dientes por lado, dientes mucronados, con espínulas de 1,32,5 mm long.; nerviación dicotómica abierta, poco visible en el haz y marcadas en el envés; las venas secundarias de 5 a 7 por lado, poco ramificadas y con ángulo de inserción de 20 a 30 grados; pecíolo glabro, de 1-2 mm long. Flores solitarias dispuestas en la porción libre de hojas de los braquiblastos; pedicelo rojizo, granular-pubérulo, de 5-9 mm long.; sépalos amarillentos con nervios muy notables y dispuestos en 2 a 4 series y en tamaño creciente; los exteriores más pequeños y con una línea dorsal rojiza, los interiores de menor o igual longitud que los pétalos pero notablemente más angostos $(1: 1,8-2,2)$. Pétalos 5 , amarillo-anaranjados, obovados u orbiculares (1:1,3-1,6), de 6-7,5 mm long. x 4-5,5 mm lat., ápice redondeado; nervaduras muy notables, las laterales dicotómicamente ramificadas sub-basalmente y la central simple o cortamente ramificada en el ápice; glándulas sub-basales, fusiformes, de 1,5 mm long. x 0,5 mm lat. Estambres 5, de 4,5-5,5 mm long., ápice espatulado-truncado y levemente producido; anteras de menos de la mitad de la longitud total del estambre. Ovario lageniforme, de 4,2-4,8 mm long.; óvulos 5 ó 6, subsésiles y de inserción basal. Fruto negro-azulado, seríceo, de 6-8 mm long. x 4,5-6 mm lat. Estilo + estigma de 1,2-1,6 mm long. Semillas 2 a 4 (raro más), naviculares, superficie foveolada y castaño-oscura en material seco.
Distribución y hábitat. Habita las montañas húmedas del noroeste de Argentina y sur de Bolivia, por arriba de los $3000 \mathrm{~m}$, en vegetación dominada por pastizales.

Aspectos ecológicos y fenología. Es un arbusto achaparrado que raramente sobrepasa un metro de altura. Crece solitario o en pequeños grupos, en faldeos graminosos o roquedales en el nivel de los pastizales de neblina. Florece entre octubre y diciembre y la fructificación se extiende hasta marzo.

Categoría en la Lista Roja de IUCN (2001). Debido a que esta especie tiene un estrecho rango de distribución altitudinal, y habita ambientes sometidos a eventuales incendios, debe ser considerada como Vulnerable $(\mathrm{Vu})$.

Etimología. El epíteto calilehua, vocablo compuesto de origen aymara, hace referencia al nombre de las sierras de donde proviene el tipo de la especie.

\section{Observaciones}

1. Berberis calilehua tiene similitudes morfológicas con especies andinas del subgrupo Aequinocciales, secciones Confertae Ahrendt y Virgatae Schneider (sensu Ahrendt, 1961). Algunas de estas especies, tales como B. lutea Ruiz et Pav., $B$. phyllacantha Rusby, B. conferta Kunth, tienen marcadas semejanzas entre sí, lo que torna confusa y controversial su delimitación taxonómica, tal que algunos autores les reconocen el status de especies (Ahrendt, 1961), mientras que otros las consideran jerárquicamente subordinadas (Macbride, 1938) e incluso sinónimas. Tal es así que muchas de las especies reconocidas para Perú y Bolivia (Schneider, 1908; Macbride, 1938; Ahrendt, 1961), en el Catálogo de Angiospermas y Gimnospermas del Perú (Liesner, 1993) fueron consideradas, a sugerencia de D.N. Smith, "with comments regarding synonymy from the late D.N. Smith (MO)", como sinónimos de Berberis lutea. Sin embargo no existe ningún respaldo bibliográfico que sostenga este criterio y hasta es probable que esta simplificación haya aumentado la confusión en la clasificación de estas especies. Esto se trasluce al revisar especímenes depositados en los diferentes herbarios, muchos de los cuales 
presentan re-determinaciones realizadas por un mismo especialista en años sucesivos, incluso dándose el caso de aplicarse nombres distintos a especímenes duplicados.

Es evidente que algunas de las especies de estas secciones (Confertae y Virgatae) presentan problemas para su delimitación, lo que probablemente se deba a que muchos de los caracteres empleados para tal fin parecen no ser muy constantes o pueden responder al hábitat, edad de la planta, época de la colecta e incluso a la posición en el individuo mismo. Tal es el caso de la pubescencia y color del tallo y/o ramas y la longitud y pubescencia del pedicelo de la flor o fruto. El carácter flor solitaria o en fascículos también puede ser discutido ya que en algunos casos las flores solitarias se disponen muy próximas entre si, dando el aspecto de un fascículo poco numeroso.

2. Las espinas en B. calilehua son pequeñas, foliáceas, de simples a 5 (-7)-fidas y su presencia depende de la edad de las ramas que se consideren; en las jóvenes las espinas son conspicuas en la base de los braquiblastos, mientras que en las ramas más viejas están ausentes o en proceso de descomposición.

3. Del análisis de los protólogos y de imágenes de alta resolución de los ejemplares tipo de las especies afines surge que Berberis calilehua difiere de $B$. lutea por las flores solitarias, hojas de mayor tamaño (13-25 x 7-11 mm vs. 10-20 x 5-9 mm), marcadamente espinoso-dentadas y estambres espatulado-truncados; de B. phyllacantha. y $B$. carinata Lechl. por la nerviación foliar dicotómica abierta; de $B$. rusbyana Ahrendt por el mayor tamaño de las hojas (8-15 x 5-8 mm) y margen espinoso-dentado; de B. saxícola Lechl. por el menor tamaño (30-60 x $25 \mathrm{~mm}$ ), margen y tipo de nerviación de las hojas y de $B$. rariflora Lechl. por el tamaño (10-18 x 3-5 mm) y forma de las hojas y fruto con estilo conspicuo.

4. El análisis de isotipos depositados en el Herbario LIL (Bang 1614 y Steinbach 9874) nos permite sostener como válidas a $B$. phyllacantha y a B. rusbyana, especies del centro-norte de Bolivia, reportadas como sinónimas (Ulloa Ulloa, 2014).

5. Una especie de Berberis, pero con otro nombre (B. rariflora), fue citada como novedad para las serranías de Calilegua (Orsi et al., 1987). Aunque no vimos el mencionado material, por la zona y por el hábitat descripto es probable que se haya tratado de una determinación errónea de la especie aquí referida.

Material estudiado. ARGENTINA. Prov. Jujuy: Dpto. Ledesma, ladera este del Cerro Hermoso, 3450 m s.m., 3-X-2008, Bulacio \& Ayarde 1298 (LIL). Dpto. Valle Grande, faldeo noroeste del Cerro Hermoso, 3250 m s.m., 20-I-1996, Ayarde 537 (LIL). Prov. Salta: Dpto. Santa Victoria, Los Toldos, cuesta de San José, 3200-3500 m s.m., 7-II-1953, Sleumer 3827 (LIL). BOLIVIA. Dpto. Potosí: Prov. Charcas, de Ocurí hacia Pajri cuchu, 3940 m s.m., 12-III-1993, Torrico \& Peca 116 (LPB).

Se propone la siguiente clave para separar especies andinas próximas entre si, la cual se basa principalmente en caracteres macroscópicos fácilmente discernibles. Entre éstos, los relacionados a la morfología foliar (nerviación y tipo de margen), los que en Berberis tienen un fuerte control genético y filogenético (Pavón-Mora \& González, 2012).

1. Flores en fascículo. Hojas con margen entero o tricuspidadas.

B. lutea

1 '. Flores solitarias. Hojas con margen entero a espinoso-sinuado.

2. Hojas lineales, margen entero.

B. rariflora

2'. Hojas elípticas a obovadas, margen espinoso-sinuado.

3. Nerviación divaricada, cerrada.

4. Hojas sinuadas en todo el margen, elípticas a ovadas, de más de $30 \mathrm{~mm}$ long.

4'. Hojas sinuadas hacia el ápice, obovadas, de menos de $25 \mathrm{~mm}$ long.

5. Margen serrado, hojas angostamente obovadas $(1: 2,2-2,6)$.

B. saxicola

5 '. Margen dentado, hojas anchamente obovadas $(1: 1,6-2,0)$.

B. phyllacantha

3'. Nerviación dicotómica, abierta.

6. Braquiblastos muy cortos o ausentes, hojas margen serrado. B. carinata

6'. Braquiblastos conspicuos, hojas margen dentado. 


\section{Agradecimientos}

A la Fundación Miguel Lillo por la provisión de fondos para este estudio, a los curadores de los herbarios LIL, SI, CORD, LPB, BOLV, HSB, USZ, a la Srta. Lelia Bordón y al Sr. Alberto Gutiérrez, de la Sección Iconografía de la Fundación Miguel Lillo y a los revisores, por sus oportunas y valiosas observaciones.

\section{Bibliografía}

AHRENDT, L. W. A. 1961. Berberis and Mahonia. A taxonomic revision. Journ. Linn. Soc. (Bot.) 57: 1-369.

IUCN, 2001. IUCN Red List Categories and Criteria, Version 3.1. Prepared by the IUCN Species Survival Commission. IUCN, Gland, Switzerland and Cambridge, UK.

LIESNER, R. B. 1993. Berberis (Berberidaceae). En: Brako, L. \& J. L. Zarucchi (eds.), Catálogo de las Angiospermas y Gimnospermas del Perú, Monogr. Syst. Bot. Missouri Bot. Gard. 45: 196-198.

MACBRIDE, J. F. 1938. Berberis (Berberidaceae). Flora of Peru, Field Mus. Nat. Hist. (Bot.) 13, 2 (3): 665680.

ORSI, M. C. 1976. Sinopsis de las especies argentinas del género Berberis (Berberidaceae). Bol. Soc. Argent. Bot. 17 (1-2): 127-149.
ORSI, M. C., C. A. IUDICA \& E. D. RAMADORI. 1987. Berberis rariflora Lechler (Berberidaceae), nueva especie para la Argentina. Resúmenes 21 Jornadas Argentinas de Botánica: 45. Santiago del Estero.

PAVÓN-MORA, N. \& F. GONZÁLEZ. 2012. Leaf development, metamorphic heteroblasty and heterophylly in Berberis s. l. (Berberidaceae). Bot. Rev. 78: 463-489.

SCHNEIDER, C. K. 1908. Berberides andinae. En: Engler, A., Bot. Jahrb. Syst. 42: 81-85.

ULlOA ULlOA, C. 2014. Berberis (Berberidaceae). En: Catálogo de las plantas vasculares de Bolivia. Tropicos.org. Missouri Botanical Garden. Disponible en: http://www.tropicos.org/Name/42000031. Acceso 26 mayo 2015.

ZULOAGA, F. O. \& O. MORRONE. 1999. Berberis (Berberidaceae). En: Zuloaga F. O. \& O. Morrone (eds.), Catálogo de las plantas vasculares de Argentina 2. Monogr. Syst. Bot. Missouri Bot. Gard. 74: 357-361.

ZULOAGA, F. O., O. MORRONE \& M. J. BELGRANO (eds.) Berberis (Berberidaceae). En: Catálogo de las plantas vasculares del Cono Sur. Instituto de Botánica Darwinion. Disponible en: http://www. darwin.edu.ar/Proyectos/FloraArgentina/FA.asp. Acceso 26 mayo 2015.

Recibido el 4 de abril de 2015, aceptado el 1 de junio de 2015 . 